\title{
A Social Choice Analysis of the Borda Rule in a General Linguistic Framework
}

\author{
José Luis García-Lapresta, Bonifacio Llamazares, Miguel Martínez-Panero \\ PRESAD Research Group, Department of Applied Economics, University of Valladolid, \\ Avda. Valle de Esgueva 6, \\ Valladolid, 47011, Spain \\ E-mail:\{lapresta,boni,panero\}@eco.uva.es \\ Received: 15-01-2010 \\ Accepted: 25-05-2010
}

\begin{abstract}
In this paper the Borda rule is extended by allowing the voters to show their preferences among alternatives through linguistic labels. To this aim, we need to add them up for assigning a qualification to each alternative and then to compare such qualifications. Theoretically, all these assessments and comparisons fall into a totally ordered commutative monoid generated by the initial set of linguistic labels. Practically, we show an example which illustrates the suitability of this linguistic approach. Finally, some interesting properties for this Borda rule are proven in the Social Choice context.
\end{abstract}

Keywords: Linguistic preferences, Borda rule, Social Choice, Voting systems, Computing with words.

\section{Introduction}

There is a wide variety of group decision making methods with particular good features (see Dummet $^{1}$, Nurmi ${ }^{2}$, Fishburn ${ }^{3}$ and Saari $^{4}$, among others). However, it happens that different procedures may provide distinct outcomes. An extreme perplexing case of this divergency appears in Malkevitch $^{5}$ : a profile is shown where each of all five possible alternatives becomes the winner under different well-known voting systems. Taking this fact into account, the use of appropriate methods for making collective decisions in each specific situation must firstly rely on a faithful and complete reflect of voters' opinions, so that the collective results will generate as little opposition as possible.

Definitely, an objectionable method is that of plurality, where only the most desired alternative is chosen by each voter, being the winner determined by the greatest total amount of votes. As pointed out early in 1770 by Borda ${ }^{6}$, this procedure might select an alternative which is the worst for more than half the electorate. This undesirable effect is due to the little part of the information that voters can provide which is processed by this method: only their best alternatives are considered, but not their opinion about the others. However, in spite of this criticism, plurality rule is still used in many elections (see Saari ${ }^{4}$ ).

Positional voting systems, where the outcomes are determined by rankings of alternatives (see Gärdenfors ${ }^{7}$ and $\operatorname{Riker}^{8}$ ), and methods based on pairwise comparisons of alternatives by the voters, are closer to voters' opinions than those which only aggregate the "iceberg top", as plurality rule does.

Among such voting systems, the Borda count is one of the best known and valuable procedures. In this way, with some reservations, Dummett ${ }^{9}$ considers the Borda rule as

the best tool for reaching the decision most likely 
to be correct when the object is to reconcile different judgements about effective means to a common aim, and the most equitable method of determining a resultant of divergent desires.

Analogously, "the Borda count appears to be optimal" according to Saari ${ }^{10}$.

Borda's original scheme (which we call throughout this paper classic Borda rule) assumes that the voters arrange all the alternatives in a linear manner, so that each alternative will be scored as the number of alternatives worse than that considered, being winner(s) those alternative(s) with the greatest total score. On forerunners of the Borda count and historical analyses of this method, see Black ${ }^{11}$ and McLean and Urken ${ }^{12}$. In order to generalize this point of view, in previous papers fuzzy preferences in voters' pairwise comparisons of alternatives have been considered. In this way, Marchant ${ }^{13,14}$ and García-Lapresta and Martínez-Panero ${ }^{15,16}$ have presented extensions of the Borda rule where voters can show indifference or show preference through numbers in the unit interval. However, there is a widespread agreement in the fact that voters tend to show their preferences in a linguistic manner rather than in an accurate numerical way (see Zadeh ${ }^{17,18}$ ). For this reason, in García-Lapresta et al. ${ }^{19,20}$, different variants of the Borda rule have been considered by allowing the voters to express several preference modalities through linguistic labels (totally, highly, rather, slightly, etc.), as happening in real life.

Here we also use linguistic terms to reflect different levels of preference, but in this paper we consider an abstract framework. Anyway, whatever the chosen objects to represent different degrees of preference will be, they ought to be added up, and the obtained results ought to be ordered. There is not a standard way to do this. According to Herrera et $a l .{ }^{21}$ :

Two main different approaches are used to aggregate and compare linguistic values: the first acts by direct computation on labels [...]; and the second uses the associated membership functions. Most of the available techniques belong to the latter. However, the final results of these are fuzzy sets which do not correspond to any label in the original term set. To obtain a label, a "linguistic approximation" is needed.

Nevertheless, the linguistic approximation is not free of problems, such as the loss of information due to linguistic computations (see, for instance, Yager $^{22}$ ).

This paper follows a comprehensive formal treatment developed by García-Lapresta ${ }^{23}$ for presenting a linguistic approach to the simple majority rule, and then also successfully implemented for the Borda rule (for different purposes than those pursued in the mentioned paper) in García-Lapresta et al. ${ }^{24}$.

In the voting scheme to be devised, the voters will pairwise compare all the alternatives through previously established linguistic labels. According to Borda's voting conception, from this individual information a qualification is considered to be assigned to each alternative, corresponding to the sum of labels which compare that alternative with all the others ${ }^{\mathrm{a}}$. These opinions will be aggregated by adding individual results up, and the winner(s) will be determined as the best alternative(s) according to some previous established ordering.

The paper is organized as follows. In Section 2, as preliminaries, we consider a formalization of the classic Borda count and then a generalization which allows the voters to show indifference among distinct alternatives; in addition, we also introduce linguistic preferences which allow us to extend such score-based Borda methods to a linguistic framework. In Section 3 the aforementioned linguistic Borda rule is defined and an example of their implementation is shown and the obtained results are comparatively related to the classic approach. Section 4 is devoted to translate some well-known Social Choice-type properties to a linguistic point of view and to check the fulfillment of them by the devised Borda rule. Finally, Section 5 concludes with some considerations.

\footnotetext{
${ }^{\text {a In García-Lapresta et al. }}{ }^{24}$, another possibility founded in the classic Borda count has been taken into account. For each voter and alternative, it consists in adding up only those labels corresponding to worse alternatives than that considered to be valued. However, the results obtained in the mentioned paper for this restricted case have lead us to deal only with the approach followed in the present paper, because this generalization satisfies better properties than those of the restricted case.
} 


\section{Preliminaries}

Let $X=\left\{x_{1}, x_{2}, \ldots, x_{n}\right\}$ be a finite set of alternatives, with $n \geqslant 2$, and $m$ voters, with $m \geqslant 2$. In order to extend the usual representation of ordinary preferences to a linguistic context, we will use the following matrix for each voter $k$ :

$$
M_{k}=\left(\begin{array}{cccc}
r_{11}^{k} & r_{12}^{k} & \cdots & r_{1 n}^{k} \\
r_{21}^{k} & r_{22}^{k} & \cdots & r_{2 n}^{k} \\
\cdots & \cdots & \cdots & \cdots \\
r_{n 1}^{k} & r_{n 2}^{k} & \cdots & r_{n n}^{k}
\end{array}\right)
$$

where $r_{i j}^{k}$ will codify, in a numerical or linguistic manner (as in forthcoming Subsections 2.1 and 2.2, respectively), the opinion for alternative $x_{i}$ when compared to alternative $x_{j}$.

With $\mathscr{P}(X)$ we denote the set of ordinary preference relations (asymmetric ordinary binary relations) on $X$. Given $P \in \mathscr{P}(X)$, the indifference relation associated with $P$ will be denoted by $I$, i.e., $x_{i} I x_{j}$ if neither $x_{i} P x_{j}$ nor $x_{j} P x_{i}$. We denote $P \cup I$ the ordinary preference-indifference relation defined by

$$
x_{i}(P \cup I) x_{j} \Leftrightarrow\left(x_{i} P x_{j} \text { or } x_{i} I x_{j}\right) .
$$

A linear order is a preference structure where $P$ is transitive and $x_{i} I x_{j}$ only whenever $x_{i}=x_{j}$. On the other hand, $P$ is negatively transitive if $P \cup I$ is a weak order or, equivalently, both $P$ and $I$ are transitive.

\subsection{The classic Borda count}

The classic Borda count requires that each voter linearly arranges the alternatives, so that every alternative is individually scored as the number of alternatives worse than that considered. In this way, the best alternative obtains $n-1$ points, the second best one obtains $n-2$ points, and so on in a descending manner, so that the worst alternative receives 0 points.

In a formal way, a linear order $P^{k}$ is shown by each voter $k, k=1, \ldots, m$, for expressing his/her preferences, and we denote by $I^{k}$ the indifference relation associated with the linear order.
The alternative $x_{i}$ obtains the individual Borda score $\#\left\{x_{j} \mid x_{i} P^{k} x_{j}\right\}$. This can be also obtained by defining the coefficients of the aforementioned matrix $M_{k}$ as

$$
r_{i j}^{k}= \begin{cases}1, & \text { if } x_{i} P^{k} x_{j}, \\ \frac{1}{2}, & \text { if } x_{i} I^{k} x_{j}, \\ 0, & \text { if } x_{j} P^{k} x_{i},\end{cases}
$$

so that voter $k$ assigns to alternative $x_{i}$ the following score:

$$
b_{k}\left(x_{i}\right)=\sum_{\substack{j=1 \\ j \neq i}}^{n} r_{i j}^{k} .
$$

If we introduce the count

$$
r_{k}\left(x_{i}\right)=\sum_{j=1}^{n} r_{i j}^{k}
$$

where the exception $j \neq i$ corresponding to the only appearing indifference is avoided (i.e., that of $x_{i}$ with itself), the following relationship trivially holds:

$$
r_{k}\left(x_{i}\right)=b_{k}\left(x_{i}\right)+\frac{1}{2} \text {. }
$$

This new count provides a rank of individual scores, from best to worst, as follows:

$$
\left\{n-\frac{1}{2}, n-\frac{3}{2}, \ldots, \frac{3}{2}, \frac{1}{2}\right\} .
$$

Notice that this spectrum allows an alternative way to define the Borda rule, because it is just a translation of the set of individual classic Borda scores.

In this way, by considering the matrix $M_{k}$, the classic Borda count $b_{k}\left(x_{i}\right)$ adds all the entries of row $i$ except for $r_{i i}^{k}$, while $r_{k}\left(x_{i}\right)$ adds all the coefficients in that row.

Adding the individual scores up, collective scores are defined for each alternative as

$$
\begin{aligned}
& \bar{b}\left(x_{i}\right)=\sum_{k=1}^{m} b_{k}\left(x_{i}\right), \\
& \bar{r}\left(x_{i}\right)=\sum_{k=1}^{m} r_{k}\left(x_{i}\right) .
\end{aligned}
$$

Obviously,

$$
\bar{r}\left(x_{i}\right)=\bar{b}\left(x_{i}\right)+\frac{m}{2} .
$$


Hence, again, the scores under these two schemes are related through a translation. The most collective scored alternative(s) under any of these schemes will become the winner(s). In fact, a collective negatively transitive preference relation can be obtained from any of these (equivalent) approaches as

$$
x_{i} P^{B} x_{j} \Leftrightarrow \bar{b}\left(x_{i}\right)>\bar{b}\left(x_{j}\right) \Leftrightarrow \bar{r}\left(x_{i}\right)>\bar{r}\left(x_{j}\right) .
$$

The classic Borda count (intended only for linear orders) can be generalized to weak orders, i.e., when indifference among distinct alternatives are allowed to be shown by the voters (see Black ${ }^{25}$ and Gärdenfors $^{7}$ ). In such situation, coefficients $1 / 2$ may appear in $M_{k}$ other than those standing on its principal diagonal, but it is worth mentioning that the equivalences between the counts $b_{k}$ and $r_{k}$, and also between $\bar{b}$ and $\bar{r}$, do not hold in this case. What we aim here is another advance: to devise a Borda count when voters' preferences on alternatives are shown in a linguistic fashion.

\subsection{Linguistic preferences}

Since ordinary preference relations (like linear orders considered under the classic Borda count) are too rough for capturing individual opinions, we consider another possible approach, more flexible and closer to voters' way of thinking than the above mentioned model (see Zadeh ${ }^{18}$ ). In this way, voters will be allowed to declare their preferences between each pair of alternatives in a linguistic manner, namely: one alternative is preferred to the other (absolutely or somewhat -in several allowed degrees-), or both alternatives are indifferent.

Along the paper we consider a set of linguistic labels $L=\left\{l_{0}, l_{1}, \ldots, l_{s}\right\}$, with $s \geqslant 2$, ranked by a linear order $<$ on $L: l_{0}<l_{1}<\cdots<l_{s}$. Suppose that there is an intermediate label representing indifference, and the rest of labels are defined around it symmetrically. The number of labels, $s+1$, will be odd and, consequently, $l_{s / 2}$ is the central label.

The fact that for each linguistic label in $L$ there exists another linguistic label in a symmetric position with respect to the central label can be explained by means of the negation operator $N: L \longrightarrow L$ de- fined by $N\left(l_{i}\right)=l_{s-i}$ for every $i \in\{0,1, \ldots, s\}$. Notice that $N\left(l_{s / 2}\right)=l_{s / 2}, N\left(N\left(l_{i}\right)\right)=l_{i}$ and $l_{i}<l_{j} \Leftrightarrow$ $N\left(l_{j}\right)<N\left(l_{i}\right)$, for all $i, j \in\{0,1, \ldots, s\}$.

Remark 1. Negation functions (or operators) in sets of linguistic labels have become standard in the literature (see, for example, Yager ${ }^{26}$, Torra ${ }^{27}$ and the references therein). They can be defined by involutiveness and order-reversing conditions, or, alternatively, as symmetrical operators (we follow this way). However, according to Torra 27 "negation functions when considered in relation to linguistic labels can be understood as antonyms (Soto and Trillas $^{28}$ )". And in fact, there are authors who use "antonym operators" exactly in the same sense that we do with "negation operators" (see Kacprzyk et $\left.a l .{ }^{29}\right)$.

Definition 1. A linguistic preference relation on $X$ based on $L$ is a $L$-valued binary relation $R: X \times X \longrightarrow L$ satisfying $r_{j i}=N\left(r_{i j}\right)$ for all $x_{i}, x_{j} \in X$, where $r_{i j}=R\left(x_{i}, x_{j}\right)$.

We denote by $\mathscr{R}_{L}(X)$ the set of linguistic preference relations on $X$ based on $L$. Notice that $r_{i i}=l_{s / 2}$ for every $i \in\{1, \ldots, n\}$ whenever $R \in \mathscr{R}_{L}(X)$.

It is worth noting that the condition appearing in Definition 1 is related to asymmetry property of ordinary preferences and reciprocity axiom of fuzzy preferences (on this last case, see, for instance, Nurmi $^{30}$ and García-Lapresta and Llamazares ${ }^{31}$ ).

The linguistic preference relation for each voter $k, R^{k}$, can be captured through the already considered matrix

$$
M_{k}=\left(\begin{array}{cccc}
r_{11}^{k} & r_{12}^{k} & \cdots & r_{1 n}^{k} \\
r_{21}^{k} & r_{22}^{k} & \cdots & r_{2 n}^{k} \\
\cdots & \cdots & \cdots & \cdots \\
r_{n 1}^{k} & r_{n 2}^{k} & \cdots & r_{n n}^{k}
\end{array}\right)
$$

whose entries now become linguistic labels and symmetric coefficients with respect to the main diagonal are also symmetric around the central label.

According to the Borda rule conception, individual opinions expressed through linguistic labels must be aggregated, and the winner must be determined as the best alternative according to a previously established ordering. So, labels have to be added, and the outcomes ought to be compared. 
This is the reason why we consider the commutative monoid $(\langle L\rangle,+)$ generated by $L$ through all possible sums of labels of $L$ with an associative and commutative binary operation + on $\langle L\rangle$ :

(i) $L \subset\langle L\rangle$

(ii) $l+l^{\prime} \in\langle L\rangle$, for all $l, l^{\prime} \in\langle L\rangle$

(iii) $l+\left(l^{\prime}+l^{\prime \prime}\right)=\left(l+l^{\prime}\right)+l^{\prime \prime}$, for all $l, l^{\prime}, l^{\prime \prime} \in\langle L\rangle$

(iv) $l+l^{\prime}=l^{\prime}+l$, for all $l, l^{\prime} \in\langle L\rangle$

(v) There exists $l^{*} \in\langle L\rangle$ (neutral element) such that $l+l^{*}=l$, for every $l \in\langle L\rangle$.

In addition to this, $\langle L\rangle$ is considered to be endowed with a total order $\leqslant$ which is compatible with the former order on $L$ :

(vi) $l \leqslant l$, for every $l \in\langle L\rangle$

(vii) $\left(l \leqslant l^{\prime}\right.$ and $\left.l^{\prime} \leqslant l\right) \Rightarrow l=l^{\prime}$, for all $l, l^{\prime} \in\langle L\rangle$

(viii) $\left(l \leqslant l^{\prime}\right.$ and $\left.l^{\prime} \leqslant l^{\prime \prime}\right) \Rightarrow l \leqslant l^{\prime \prime}$, for all $l, l^{\prime}, l^{\prime \prime} \in\langle L\rangle$

(ix) $l \leqslant l^{\prime}$ or $l^{\prime} \leqslant l$, for all $l, l^{\prime} \in\langle L\rangle$

(x) $l_{0}<l_{1}<\cdots<l_{s}$, where $<$ is the strict order associated with $\leqslant\left(l<l^{\prime}\right.$ if $l \leqslant l^{\prime}$ and $l \neq l^{\prime}$, for all $\left.l, l^{\prime} \in\langle L\rangle\right)$.

We also assume the following compatibility property:

(xi) $l<l^{\prime} \Rightarrow l+l^{\prime \prime}<l^{\prime}+l^{\prime \prime}$, for all $l, l^{\prime}, l^{\prime \prime} \in\langle L\rangle$.

So, $(\langle L\rangle,+, \leqslant)$ is a totally ordered commutative monoid (see García-Lapresta ${ }^{23}$, where $l^{*}=l_{0}$ is taken).

We note that for each $l \in\langle L\rangle$, there exist integers $\lambda_{0}, \ldots, \lambda_{s} \geqslant 0$ such that $l=\sum_{i=0}^{s} \lambda_{i} l_{i}$, where $\lambda_{i} l_{i}$ is the addition of $\lambda_{i}$ times the label $l_{i}$, and $0 l_{i}=l^{*}$.

As pointed out before, the original order considered in $L$ becomes reversed by the action of the negation operator. The following definition characterizes the class of totally ordered monoids satisfying an extension of such reversal property, as shown in Proposition 1.

Definition 2. A totally ordered commutative monoid $(\langle L\rangle,+, \leqslant)$ on a set of linguistic labels $L=$ $\left\{l_{0}, l_{1}, \ldots, l_{s}\right\}$ is well-balanced if

$$
l_{i}+N\left(l_{i}\right)=l_{j}+N\left(l_{j}\right)
$$

for all $i, j \in\{0,1, \ldots, s\}$.

Proposition 1. Let $(\langle L\rangle,+, \leqslant)$ be a totally ordered commutative monoid on a set of linguistic labels $L=\left\{l_{0}, l_{1}, \ldots, l_{s}\right\}$. The following statements are equivalent:

1. $(\langle L\rangle,+, \leqslant)$ is well-balanced.

2. $l_{i}+N\left(l_{i}\right)=2 l_{s / 2}$ for every $i \in\{0, \ldots, s\}$.

$$
\begin{aligned}
& \text { 3. } \sum_{i=0}^{s} \lambda_{i} l_{i}>\sum_{i=0}^{s} \mu_{i} l_{i} \Leftrightarrow \sum_{i=0}^{s} \lambda_{i} N\left(l_{i}\right)<\sum_{i=0}^{s} \mu_{i} N\left(l_{i}\right) \\
& \text { whenever } \sum_{i=0}^{s} \lambda_{i}=\sum_{i=0}^{s} \mu_{i} .
\end{aligned}
$$

\section{Proof.}

$1 \Rightarrow$ 2: Obvious.

$2 \Rightarrow 3$ : Provided that $N\left(N\left(l_{i}\right)\right)=l_{i}$ for every $i \in\{0,1, \ldots, s\}$, it is sufficient to prove that

$$
\sum_{i=0}^{s} \lambda_{i} l_{i}>\sum_{i=0}^{s} \mu_{i} l_{i} \Rightarrow \sum_{i=0}^{s} \lambda_{i} N\left(l_{i}\right)<\sum_{i=0}^{s} \mu_{i} N\left(l_{i}\right)
$$

whenever $\sum_{i=0}^{s} \lambda_{i}=\sum_{i=0}^{s} \mu_{i}$. This is proven by contradiction. Suppose that

$$
\sum_{i=0}^{s} \lambda_{i} l_{i}>\sum_{i=0}^{s} \mu_{i} l_{i} \text { and } \sum_{i=0}^{s} \lambda_{i} N\left(l_{i}\right) \geqslant \sum_{i=0}^{s} \mu_{i} N\left(l_{i}\right) .
$$

Then, on the one hand,

$$
\sum_{i=0}^{s} \lambda_{i} l_{i}+\sum_{i=0}^{s} \lambda_{i} N\left(l_{i}\right)>\sum_{i=0}^{s} \mu_{i} l_{i}+\sum_{i=0}^{s} \mu_{i} N\left(l_{i}\right)
$$

but, on the other hand

$$
\begin{aligned}
\sum_{i=0}^{s} \lambda_{i} l_{i}+\sum_{i=0}^{s} \lambda_{i} N\left(l_{i}\right) & =\sum_{i=0}^{s} \lambda_{i}\left(l_{i}+N\left(l_{i}\right)\right) \\
& =\sum_{i=0}^{s} \lambda_{i}\left(2 l_{s / 2}\right) \\
& =2\left(\sum_{i=0}^{s} \lambda_{i}\right) l_{s / 2}
\end{aligned}
$$


and

$$
\begin{aligned}
\sum_{i=0}^{s} \mu_{i} l_{i}+\sum_{i=0}^{s} \mu_{i} N\left(l_{i}\right) & =\sum_{i=0}^{s} \mu_{i}\left(l_{i}+N\left(l_{i}\right)\right) \\
& =\sum_{i=0}^{s} \mu_{i}\left(2 l_{s / 2}\right) \\
& =2\left(\sum_{i=0}^{s} \mu_{i}\right) l_{s / 2}
\end{aligned}
$$

i.e., a contradiction because $\sum_{i=0}^{s} \lambda_{i}=\sum_{i=0}^{s} \mu_{i}$.

$3 \Rightarrow 1$ : This is proven by contradiction. Suppose there exist $i, j \in\{0,1, \ldots, s\}$ such that $l_{i}+N\left(l_{i}\right) \neq$ $l_{j}+N\left(l_{j}\right)$. If $l_{i}+N\left(l_{i}\right)<l_{j}+N\left(l_{j}\right)$ - the case $l_{i}+N\left(l_{i}\right)>l_{j}+N\left(l_{j}\right)$ can be studied in a similar way- then $N\left(l_{i}\right)+N\left(N\left(l_{i}\right)\right)>N\left(l_{j}\right)+N\left(N\left(l_{j}\right)\right)$, or equivalently, $N\left(l_{i}\right)+l_{i}>N\left(l_{j}\right)+l_{j}$; i.e., a contradiction.

\subsection{The well-balancedness condition on trapezoidal fuzzy numbers}

Let us show the implications of the wellbalancedness condition when trapezoidal fuzzy numbers (TFNs) are used to represent linguistic labels. This is a relevant question because TFNs are a suitable and widely used tool in order to capture vagueness in voters' assessments (see Delgado et $a l .{ }^{32}$, among others). In addition, we note that TFNs generalize other well-known types of models very used in the literature for dealing with linguistic labels, such as real numbers, intervals and triangular fuzzy numbers.

Given four real numbers $a, b, c, d$ such that $a \leqslant b \leqslant c \leqslant d$, the TFN $t=(a, b, c, d)$ is defined by its membership function $\mu_{t}: \mathbb{R} \longrightarrow[0,1]$, where

$$
\mu_{t}(x)=\left\{\begin{array}{l}
0, \text { if } x<a \text { or } x>d, \\
\frac{x-a}{b-a}, \text { if } a<x<b \\
1, \text { if } b \leqslant x \leqslant c \\
\frac{d-x}{d-c}, \text { if } c<x<d
\end{array}\right.
$$

and

$\mu_{t}(a)=\left\{\begin{array}{l}0, \text { if } a<b, \\ 1, \text { if } a=b,\end{array} \quad \mu_{t}(d)= \begin{cases}0, & \text { if } d>c \\ 1, & \text { if } d=c\end{cases}\right.$

As its name suggests, the graphic representation of a TFN is just a trapezoid (see Figure 1).

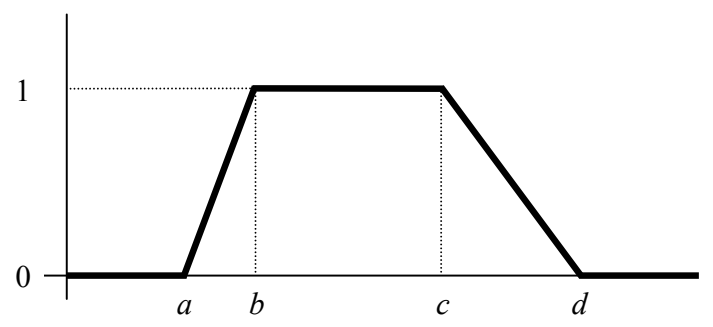

Fig. 1. Trapezoidal fuzzy number.

In this subsection we identify each linguistic label $l \in L$ with a fixed TFN, namely, $l=(a, b, c, d)$, with $0 \leqslant a \leqslant b \leqslant c \leqslant d \leqslant 1$. For computation purposes, there is a univocal componentwise way for defining the addition of TFNs (see, for instance, Dubois and $\operatorname{Prade}^{33}$ ):

$$
\begin{aligned}
& (a, b, c, d)+\left(a^{\prime}, b^{\prime}, c^{\prime}, d^{\prime}\right) \\
& \quad=\left(a+a^{\prime}, b+b^{\prime}, c+c^{\prime}, d+d^{\prime}\right) .
\end{aligned}
$$

As for the negation operator ${ }^{a}$ we define $N(a, b, c, d)=(1-d, 1-c, 1-b, 1-a)$.

With these assumptions, as the central label satisfies $N\left(l_{s / 2}\right)=l_{s / 2}$, it is easy to check that

$$
l_{s / 2}=\left(a_{s / 2}, b_{s / 2}, 1-b_{s / 2}, 1-a_{s / 2}\right),
$$

\footnotetext{
${ }^{a}$ When we identify a label $l$ with a TFN, $N(l)$ is not the complement of $l$ given by the membership function $\mu_{N(l)}(x)=1-\mu_{l}(x)$, but its symmetrical TFN with respect to the straight line $x=0.5$.
} 
with $0 \leqslant a_{s / 2} \leqslant b_{s / 2} \leqslant 0.5$. This means that the central label is symmetrical with respect to the straight line $x=0.5$.

Let us now take any label $l_{i}=\left(a_{i}, b_{i}, c_{i}, d_{i}\right) \in L$. Since $N\left(l_{i}\right)=\left(1-d_{i}, 1-c_{i}, 1-b_{i}, 1-a_{i}\right)$, by item 2 of Proposition 1, the well-balancedness condition implies

$$
\begin{array}{r}
\left(a_{i}+1-d_{i}, b_{i}+1-c_{i}, c_{i}+1-b_{i}, d_{i}+1-a_{i}\right) \\
=\left(2 a_{s / 2}, 2 b_{s / 2}, 2\left(1-b_{s / 2}\right), 2\left(1-a_{s / 2}\right)\right),
\end{array}
$$

and, hence

$$
d_{i}-a_{i}=1-2 a_{s / 2}, \quad c_{i}-b_{i}=1-2 b_{s / 2},
$$

for every $i \in\{0,1, \ldots, s\}$.

Geometrically, such conditions mean that all upper bases (kernels) of the TFNs representing labels in a well-balanced context must share the same length, and also the same property holds for the lower bases (supports).

In order to illustrate the previous comments, Table 1 and Figure 2 show a well-balanced set of five linguistic labels represented by TFNs.

Table 1. An example of five well-balanced linguistic labels.

\begin{tabular}{cl}
\hline Label & \multicolumn{1}{c}{ TFN } \\
\hline$l_{0}$ & $(0,0,0.12,0.24)$ \\
$l_{1}$ & $(0.19,0.22,0.34,0.43)$ \\
$l_{2}$ & $(0.38,0.44,0.56,0.62)$ \\
$l_{3}$ & $(0.57,0.66,0.78,0.81)$ \\
$l_{4}$ & $(0.76,0.88,1,1)$ \\
\hline
\end{tabular}

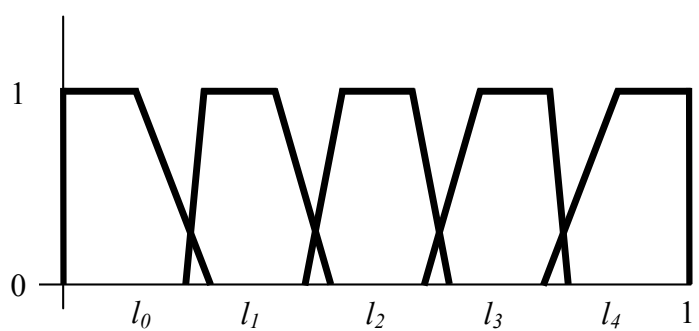

Fig. 2. Graphical representation of the previous TFNs.

We note that some authors (see, for instance, Herrera and Herrera-Viedma ${ }^{34,35}$ ) take $l_{0}=(0,0,0,0)$ and $l_{s}=(1,1,1,1)$, i.e., extreme preferences are considered to be crisp. Then, according to the previous comment, if the wellbalancedness condition holds, all the labels should be represented by TFNs collapsing to real numbers, i.e., $a_{i}=b_{i}=c_{i}=d_{i}$. Thus, in order to preserve vagueness and the well-balancedness condition jointly, extreme preferences should not be represented by real numbers.

\section{Linguistic-Based Borda Rule}

This section introduces a linguistic Borda count inspired by the classic pattern. Once this linguistic Borda count defined, an example is presented in order to illustrate this procedure and comparing it with the standard (numerical) approach. No confusion arising, we will not distinguish the notation between the linguistic case and its numerical pattern.

\subsection{From the classic to the linguistic framework}

Taking into account the counts $r_{k}$ and $\bar{r}$ appearing in Subsection 2.1, we now present a natural linguistic extension of them. The generalization of $r_{k}$ adds all linguistic labels up when comparing each alternative with all others (the alternative itself included). On the other hand, the collective count $\bar{r}$ adds individual results up taking into account that $\langle L\rangle$ is endowed with an additive operation.

Definition 3. The linguistic-based Borda count of voter $k$ is given by:

$$
r_{k}\left(x_{i}\right)=\sum_{j=1}^{n} r_{i j}^{k}
$$

Next, for each voter $k$, we introduce ordinary relations associated with the corresponding linguistic preference.

Definition 4. Let $R^{k}$ be a linguistic preference relation on $X$ based on $L$.

(i) The ordinary preference relation associated with $R^{k}, \succ_{k}$, is defined by $x_{i} \succ_{k} x_{j}$ if and only if $r_{i j}^{k}>l_{s / 2}$. 
(ii) The ordinary preference-indifference relation associated with $R^{k}, \succcurlyeq_{k}$, is defined by $x_{i} \succcurlyeq_{k} x_{j}$ if and only if $r_{i j}^{k} \geqslant l_{s / 2}$.

Notice that the condition $r_{i j}^{k}>l_{s / 2}$ appearing in the previous definition is equivalent to $r_{i j}^{k}>r_{j i}^{k}$, meaning that voter $k$ somehow prefers $x_{i}$ to $x_{j}$.

For the Borda count when defined under ordinary preferences, a desirable property (which we call representativity) establishes that the individual Borda scores must respect the individual pairwise preferences, i.e., if an alternative is preferred to another one by an voter, then the individual score given to the first alternative must be greater than that obtained by the second one. This property trivially holds for the classic Borda count (namely, when voters' preferences are linear orders) and its extension to weak orders (it is easy to check that a sufficient condition for its fulfillment is the transitivity of individual preferences, see García-Lapresta and Martínez-Panero ${ }^{15}$ ). Next, we translate this property in a formal way to the introduced linguistic case.

Definition 5. Let $R^{k}$ be a linguistic preference relation. The linguistic-based Borda count $r_{k}$ is said to be representative of $R^{k}$ if

$$
x_{i} \succ_{k} x_{j} \Rightarrow r_{k}\left(x_{i}\right)>r_{k}\left(x_{j}\right)
$$

for all $x_{i}, x_{j} \in X$.

In this linguistic context, it can be shown that merely transitivity of $\succ_{k}$ associated with each $R^{k}$ does not imply representativity. In this sense, García-Lapresta et al. $^{24}$ have found a transitivitytype condition which ensures the representativity of the individual linguistic Borda count:

$$
\left(x_{i} \succcurlyeq_{k} x_{j} \text { and } x_{j} \succcurlyeq_{k} x_{p}\right) \Rightarrow r_{i p}^{k} \geqslant \max \left\{r_{i j}^{k}, r_{j p}^{k}\right\} \text {, }
$$

for all $x_{i}, x_{j}, x_{p} \in X$.

As for the numerical case, we now also add the individual results up, through the additive operation of $\langle L\rangle$, in order to obtain collective outcomes.

Definition 6. The collective linguistic-based Borda count is given by:

$$
\bar{r}\left(x_{i}\right)=\sum_{k=1}^{m} r_{k}\left(x_{i}\right)
$$

Based on the previous count, a collective ordinary preference relation on $X$ (also negatively transitive, as for the classic Borda count) can be obtained as

$$
x_{i} P^{L B} x_{j} \Leftrightarrow \bar{r}\left(x_{i}\right)>\bar{r}\left(x_{j}\right) .
$$

\subsection{An illustrative example}

Consider four voters who express their preferences over $X=\left\{x_{1}, x_{2}, x_{3}\right\}$ through linear orders as shown in Table 2.

Table 2. Voters' profile

\begin{tabular}{cccc}
\hline 1 & 2 & 3 & 4 \\
\hline$x_{1}$ & $x_{1}$ & $x_{2}$ & $x_{2}$ \\
$x_{2}$ & $x_{3}$ & $x_{3}$ & $x_{1}$ \\
$x_{3}$ & $x_{2}$ & $x_{1}$ & $x_{3}$ \\
\hline
\end{tabular}

If we use the classic Borda count, the following scores are obtained:

$$
\bar{b}\left(x_{1}\right)=5, \quad \bar{b}\left(x_{2}\right)=5, \quad \bar{b}\left(x_{3}\right)=2 .
$$

Consequently, $x_{1} I^{B} x_{2} P^{B} x_{3}$, where $I^{B}$ is the indifference relation associated with $P^{B}$. Because of the tie between the highest scored alternatives, the classic Borda rule is not able to determine a single winner. Now, assume that voters show their preferences in a more nuanced manner by means of linguistic labels whose meaning appears in Table 3.

Table 3. Meaning of the linguistic labels.

\begin{tabular}{cl}
\hline Label & \multicolumn{1}{c}{ Meaning } \\
\hline$r_{i j}=l_{0}$ & $x_{j}$ is totally preferred to $x_{i}$ \\
$r_{i j}=l_{1}$ & $x_{j}$ is highly preferred to $x_{i}$ \\
$r_{i j}=l_{2}$ & $x_{j}$ is rather preferred to $x_{i}$ \\
$r_{i j}=l_{3}$ & $x_{j}$ is slightly preferred to $x_{i}$ \\
$r_{i j}=l_{4}$ & $x_{i}$ is indifferent to $x_{j}$ \\
$r_{i j}=l_{5}$ & $x_{i}$ is slightly preferred to $x_{j}$ \\
$r_{i j}=l_{6}$ & $x_{i}$ is rather preferred to $x_{j}$ \\
$r_{i j}=l_{7}$ & $x_{i}$ is highly preferred to $x_{j}$ \\
$r_{i j}=l_{8}$ & $x_{i}$ is totally preferred to $x_{j}$ \\
\hline
\end{tabular}

According to this semantics, the matrix representations of their linguistic preferences are supposed to 
be:

$$
\begin{array}{ll}
M_{1}=\left(\begin{array}{lll}
l_{4} & l_{5} & l_{6} \\
l_{3} & l_{4} & l_{6} \\
l_{2} & l_{2} & l_{4}
\end{array}\right), & M_{2}=\left(\begin{array}{lll}
l_{4} & l_{7} & l_{6} \\
l_{1} & l_{4} & l_{2} \\
l_{2} & l_{6} & l_{4}
\end{array}\right), \\
M_{3}=\left(\begin{array}{lll}
l_{4} & l_{1} & l_{3} \\
l_{7} & l_{4} & l_{5} \\
l_{5} & l_{3} & l_{4}
\end{array}\right), & M_{4}=\left(\begin{array}{lll}
l_{4} & l_{3} & l_{5} \\
l_{5} & l_{4} & l_{5} \\
l_{3} & l_{3} & l_{4}
\end{array}\right) .
\end{array}
$$

Notice that the ordinary preference relations $\succ_{k}$ associated with $R^{k}$ related to $M_{k}$ correspond to the linear orders of the voters appearing in Table 2.

When the linguistic-based Borda rule is used, we obtain the following outcomes:

$$
\begin{aligned}
& \bar{r}\left(x_{1}\right)=l_{7}+2 l_{6}+2 l_{5}+4 l_{4}+2 l_{3}+l_{1}, \\
& \bar{r}\left(x_{2}\right)=l_{7}+l_{6}+3 l_{5}+4 l_{4}+l_{3}+l_{2}+l_{1}, \\
& \bar{r}\left(x_{3}\right)=l_{6}+l_{5}+4 l_{4}+3 l_{3}+3 l_{2} .
\end{aligned}
$$

As $l_{7}+2 l_{6}+2 l_{5}+4 l_{4}+2 l_{3}+l_{1}>l_{7}+l_{6}+3 l_{5}+$ $4 l_{4}+l_{3}+l_{2}+l_{1}$, we have $x_{1} P^{L B} x_{2}$. Consequently, $x_{2}$ will never be the linguistic-based Borda winner whatever the totally ordered monoid based on the above set of labels will be. However, from the ordinal scale provided by $L$, no relationship among these two alternatives and $x_{3}$ can be established. Thus, we are unable to determine the linguistic-based Borda winner just with the previous information. But if we consider any well-balanced monoid based on the prefixed set of labels, we have by easy computations

$$
\begin{aligned}
& \bar{r}\left(x_{1}\right)=2 l_{6}+10 l_{4}, \\
& \bar{r}\left(x_{2}\right)=2 l_{5}+10 l_{4}, \\
& \bar{r}\left(x_{3}\right)=8 l_{4}+2 l_{3}+2 l_{2} .
\end{aligned}
$$

In this case, as $2 l_{6}+10 l_{4}>2 l_{5}+10 l_{4}>8 l_{4}+$ $2 l_{3}+2 l_{2}$, we have $x_{1} P^{L B} x_{2} P^{L B} x_{3}$. Therefore, $x_{1}$ is the linguistic-based Borda winner for any wellbalanced monoid based on the considered set of labels.

It is worth mentioning that merely the wellbalancedness condition is not sufficient to break all the non decisive situations. A possibility to deal with this impasse consists in representing labels by means of appropriate mathematical objects to operate with (for example, TFNs could be a suitable choice to this purpose, as pointed out before); however, we notice that the winner might depend on the choice of such TFNs.

By the way of the previous example, we can evidently assert that the classic and the linguistic-based Borda rules are not equivalent at all, as shown by the divergency of the outcomes obtained. Of course, this is not a surprising fact, due to the input data of both procedures: while the linguistic Borda count processes all the informational background, some relevant features are avoided under the classic approach.

\section{The Results}

In order to analyze some Social Choice properties into the linguistic framework, we now translate the ordinary concept of aggregation rule to this context. To this aim, we first introduce the following set

$$
\overline{\mathscr{R}}_{L}(X)=\bigcup_{m=2}^{\infty} \mathscr{R}_{L}(X)^{m}
$$

for dealing with a variable number of voters.

Definition 7. An aggregation rule is a mapping $F: \overline{\mathscr{R}}_{L}(X) \longrightarrow \mathscr{P}(X)$ which assigns a collective ordinary preference relation to each profile of individual linguistic preferences.

In order to provide collective ordinary preferences from aggregation rules faithfully representing individual opinions, it would be necessary to require some properties within the Social Choice framework. In what follows we introduce the properties appearing in the results.

Anonymity means that the outcome provided by the aggregation rule only depends on the set of the individual opinions but not on which voters have these opinions, i.e., a symmetric treatment of voters is required.

Similarly, neutrality imposes a symmetric treatment of alternatives.

Consistency means that if an alternative defeats another one for two subsets of voters, then the winner alternative keep on being the winner for the joined set of voters. This property (also called reinforcement) has been used in different characterizations of several classic voting systems (see Young ${ }^{36}$, 
Fishburn $^{37}$ and Young and Levenglick ${ }^{38}$, among others).

Monotonicity means that an alternative does not get worse with respect to another alternative in the collective preference whenever a voter improves his/her opinion of the first alternative with respect to the second one and the rest of voters do not change their opinions on that alternatives.

Strong monotonicity means that an alternative which is collectively indifferent or preferred to a second alternative should defeat the second alternative whenever a voter improves his/her opinion of the first alternative with respect to the second one and the rest of voters do not change their opinions on that alternatives. Strong monotonicity is related to a property within the classical approach of Social Choice called positive responsiveness (see $\mathrm{May}^{39}$ ). An extension of this property within the linguistic approach has been considered in García-Lapresta ${ }^{23}$. We note that every aggregation rule satisfying strong monotonicity is also monotonic.

Reversal symmetry means that the collective preference between two alternatives is reversed when all the voters reverse their opinions over that alternatives. This property has been considered by $\mathrm{Saari}^{40}$ in another context where voters rank order the alternatives.

Unanimity means that if all the voters somewhat prefer an alternative to another one, then that alternative should be collectively preferred to the second one.

Definition 8. Let $F$ be an aggregation rule.

(i) $F$ is anonymous if for every bijection $\sigma:\{1, \ldots, m\} \longrightarrow\{1, \ldots, m\}$ and every $\left(R^{1}, \ldots, R^{m}\right) \in \overline{\mathscr{R}}_{L}(X)$ :

$$
F\left(R^{\sigma(1)}, \ldots, R^{\sigma(m)}\right)=F\left(R^{1}, \ldots, R^{m}\right) .
$$

(ii) $F$ is neutral if for every bijection $\sigma:\{1, \ldots, n\} \longrightarrow\{1, \ldots, n\}$ and all $\left(R^{1}, \ldots, R^{m}\right), \quad\left(S^{1}, \ldots, S^{m}\right) \in \mathscr{\mathscr { R }}_{L}(X)$ with $F\left(R^{1}, \ldots, R^{m}\right)=P$ and $F\left(S^{1}, \ldots, S^{m}\right)=P^{\prime}$ :

If $r_{i j}^{k}=s_{\sigma(i) \sigma(j)}^{k}$ for all $i, j \in\{1, \ldots, n\}$ and $k \in\{1, \ldots, m\}$, then $x_{i} P x_{j} \Leftrightarrow x_{\sigma(i)} P^{\prime} x_{\sigma(j)}$, for all $i, j \in\{1, \ldots, n\}$.

(iii) $F$ is consistent if for all $x_{i}, x_{j} \in X$ and all $\left(R^{1}, \ldots, R^{m}\right),\left(S^{1}, \ldots, S^{m^{\prime}}\right) \in \overline{\mathscr{R}}_{L}(X)$ with

$$
\begin{aligned}
& F\left(R^{1}, \ldots, R^{m}\right)=P, F\left(S^{1}, \ldots, S^{m^{\prime}}\right)=P^{\prime} \text { and } \\
& F\left(R^{1}, \ldots, R^{m}, S^{1}, \ldots, S^{m^{\prime}}\right)=P^{\prime \prime}: \\
& \quad \text { If } x_{i} P x_{j} \text { and } x_{i} P^{\prime} x_{j} \text {, then } x_{i} P^{\prime \prime} x_{j} .
\end{aligned}
$$

(iv) $F$ is monotonic if for all $\left(R^{1}, \ldots, R^{m}\right)$, $\left(S^{1}, \ldots, S^{m}\right) \in \overline{\mathscr{R}}_{L}(X)$, with $F\left(R^{1}, \ldots, R^{m}\right)=P$ and $F\left(S^{1}, \ldots, S^{m}\right)=P^{\prime}$, such that there exist $h \in\{1, \ldots, m\}$ and $i, j \in\{1, \ldots, n\}$ with $r_{i j}^{h}<s_{i j}^{h}, r_{p q}^{h}=s_{p q}^{h}$ whenever $\{p, q\} \neq\{i, j\}$ and $R^{k}=S^{k}$ for all $k \neq h$ :

(a) If $x_{i} P x_{j}$, then $x_{i} P^{\prime} x_{j}$.

(b) If $x_{i} I x_{j}$, then $x_{i}\left(P^{\prime} \cup I^{\prime}\right) x_{j}$.

(v) $F$ is strongly monotonic if for all $\left(R^{1}, \ldots, R^{m}\right)$, $\left(S^{1}, \ldots, S^{m}\right) \in \overline{\mathscr{R}}_{L}(X)$ with $F\left(R^{1}, \ldots, R^{m}\right)=P$ and $F\left(S^{1}, \ldots, S^{m}\right)=P^{\prime}$ such that there exist $h \in\{1, \ldots, m\}$ and $i, j \in\{1, \ldots, n\}$ with $r_{i j}^{h}<s_{i j}^{h}, r_{p q}^{h}=s_{p q}^{h}$ whenever $\{p, q\} \neq\{i, j\}$ and $R^{k}=S^{k}$ for all $k \neq h$ :

$$
\text { If } x_{i}(P \cup I) x_{j} \text {, then } x_{i} P^{\prime} x_{j} \text {. }
$$

(vi) $F$ is reversal symmetric if for all $\left(R^{1}, \ldots, R^{m}\right)$, $\left(S^{1}, \ldots, S^{m}\right) \in \overline{\mathscr{R}}_{L}(X)$ with $F\left(R^{1}, \ldots, R^{m}\right)=P$ and $F\left(S^{1}, \ldots, S^{m}\right)=P^{\prime}$ :

If $s_{i j}^{k}=N\left(r_{i j}^{k}\right)$ for all $i, j \in\{1, \ldots, n\}$ and $k \in\{1, \ldots, m\}$, then $x_{i} P x_{j} \Leftrightarrow x_{j} P^{\prime} x_{i}$, for all $i, j \in\{1, \ldots, n\}$.

(vii) $F$ is unanimous if for all $x_{i}, x_{j} \in X$ and $\left(R^{1}, \ldots, R^{m}\right) \in \overline{\mathscr{R}}_{L}(X)$ with $F\left(R^{1}, \ldots, R^{m}\right)=P$ :

If $x_{i} \succ_{k} x_{j}$ for every $k \in\{1, \ldots, m\}$, then $x_{i} P x_{j}$.

In the next result we establish that the linguisticbased Borda rule enjoys some interesting properties.

Proposition 2. For every totally ordered commutative monoid $(\langle L\rangle,+, \leqslant)$ based on a set of linguistic labels $L$, the associated linguistic-based Borda rule is anonymous, neutral, consistent and strongly monotonic. Moreover, such rule is unanimous when restricted to profiles where linguistic preference relations are associated with representative counts.

\section{Proof.}

(i) Anonymity. It is straightforward because

$$
\sum_{k=1}^{m} r_{k}\left(x_{i}\right)=\sum_{k=1}^{m} r_{\sigma(k)}\left(x_{i}\right)
$$


for all bijection $\sigma$ in $\{1, \ldots, m\}$ and $\left(R^{1}, \ldots, R^{m}\right) \in \overline{\mathscr{R}}_{L}(X)$.

(ii) Neutrality. Let $\sigma$ be a bijection in $\{1, \ldots, n\}$ and $\left(R^{1}, \ldots, R^{m}\right),\left(S^{1}, \ldots, S^{m}\right) \in \overline{\mathscr{R}}_{L}(X)$ with $F\left(R^{1}, \ldots, R^{m}\right)=P$ and $F\left(S^{1}, \ldots, S^{m}\right)=P^{\prime}$. If $r_{i j}^{k}=s_{\sigma(i) \sigma(j)}^{k}$ for all $i, j \in\{1, \ldots, n\}$ and $k \in\{1, \ldots, m\}$, then $r_{k}^{\prime}\left(x_{\sigma(i)}\right)=r_{k}\left(x_{i}\right)$ for every $k \in\{1, \ldots, m\}$. Therefore,

$$
\bar{r}^{\prime}\left(x_{\sigma(i)}\right)=\sum_{k=1}^{m} r_{k}^{\prime}\left(x_{\sigma(i)}\right)=\sum_{k=1}^{m} r_{k}\left(x_{i}\right)=\bar{r}\left(x_{i}\right) .
$$

Analogously, $\bar{r}^{\prime}\left(x_{\sigma(j)}\right)=\bar{r}\left(x_{j}\right)$ and, consequently,

$$
x_{i} P x_{j} \Leftrightarrow x_{\sigma(i)} P^{\prime} x_{\sigma(j)} \text {. }
$$

(iii) Consistency. Let two alternatives $x_{i}, x_{j} \in X$ and $\left(R^{1}, \ldots, R^{m}\right),\left(S^{1}, \ldots, S^{m^{\prime}}\right) \in \bar{R}_{L}(X)$ with $F\left(R^{1}, \ldots, R^{m}\right)=P, F\left(S^{1}, \ldots, S^{m^{\prime}}\right)=P^{\prime}$ and $F\left(R^{1}, \ldots, R^{m}, S^{1}, \ldots, S^{m^{\prime}}\right)=P^{\prime \prime}$. If $x_{i} P x_{j}$ and $x_{i} P^{\prime} x_{j}$, then $\bar{r}\left(x_{i}\right)>\bar{r}\left(x_{j}\right)$ and $\bar{r}^{\prime}\left(x_{i}\right)>\bar{r}^{\prime}\left(x_{j}\right)$. Therefore,

$$
\begin{aligned}
\bar{r}^{\prime \prime}\left(x_{i}\right) & =\bar{r}\left(x_{i}\right)+\bar{r}^{\prime}\left(x_{i}\right) \\
& >\bar{r}\left(x_{j}\right)+\bar{r}^{\prime}\left(x_{j}\right)=\bar{r}^{\prime \prime}\left(x_{j}\right),
\end{aligned}
$$

i.e., $x_{i} P^{\prime \prime} x_{j}$.

(iv) Strong monotonicity. Let $\left(R^{1}, \ldots, R^{m}\right)$, $\left(S^{1}, \ldots, S^{m}\right) \in \bar{R}_{L}(X)$, with $F\left(R^{1}, \ldots, R^{m}\right)=$ $P, F\left(S^{1}, \ldots, S^{m}\right)=P^{\prime}$, such that there exist $h \in\{1, \ldots, m\}$ and $i, j \in\{1, \ldots, n\}$ with $r_{i j}^{h}<s_{i j}^{h}, r_{p q}^{h}=s_{p q}^{h}$ whenever $\{p, q\} \neq\{i, j\}$ and $R^{k}=S^{k}$ for all $k \neq h$. If $x_{i}(P \cup I) x_{j}$, then $\bar{r}\left(x_{i}\right) \geqslant \bar{r}\left(x_{j}\right)$. Since $r_{i j}^{h}<s_{i j}^{h}$, and, consequently, $s_{j i}^{h}<r_{j i}^{h}$, we have $r_{h}^{\prime}\left(x_{i}\right)>r_{h}\left(x_{i}\right)$ and $r_{h}^{\prime}\left(x_{j}\right)<r_{h}\left(x_{j}\right)$. Moreover, $r_{k}^{\prime}\left(x_{i}\right)=r_{k}\left(x_{i}\right)$ and $r_{k}^{\prime}\left(x_{j}\right)=r_{k}\left(x_{j}\right)$ for all $k \neq h$. Therefore,

$$
\bar{r}^{\prime}\left(x_{i}\right)>\bar{r}\left(x_{i}\right) \geqslant \bar{r}\left(x_{j}\right)>\bar{r}^{\prime}\left(x_{j}\right),
$$

i.e., $x_{i} P^{\prime} x_{j}$.

(v) Unanimity. Let $\left(R^{1}, \ldots, R^{m}\right) \in \bar{R}_{L}(X)$ such that the count $r_{k}$ is representative of $R^{k}$ and $x_{i} \succ_{k} x_{j}$ for every $k \in\{1, \ldots, m\}$.
This entails $r_{k}\left(x_{i}\right)>r_{k}\left(x_{j}\right)$ for every $k \in\{1, \ldots, m\}$. Therefore,

$$
\bar{r}\left(x_{i}\right)=\sum_{k=1}^{m} r_{k}\left(x_{i}\right)>\sum_{k=1}^{m} r_{k}\left(x_{j}\right)=\bar{r}\left(x_{j}\right),
$$

i.e., $x_{i} P x_{j}$.

We now show that, in a more restrictive context, the linguistic-based Borda rule also verifies reversal symmetry.

Proposition 3. For every well-balanced totally ordered commutative monoid $(\langle L\rangle,+, \leqslant)$ on a set of linguistic labels $L=\left\{l_{0}, l_{1}, \ldots, l_{s}\right\}$, the associated linguistic-based Borda rule is reversal symmetric.

Proof. Let $\left(R^{1}, \ldots, R^{m}\right),\left(S^{1}, \ldots, S^{m}\right) \in \overline{\mathscr{R}}_{L}(X)$ with $F\left(R^{1}, \ldots, R^{m}\right)=P$ and $F\left(S^{1}, \ldots, S^{m}\right)=P^{\prime}$. If $r_{i j}^{k}=s_{j i}^{k}$ for all $i, j \in\{1, \ldots, n\}$ and $k \in\{1, \ldots, m\}$, then

$$
\begin{aligned}
x_{i} P x_{j} & \Leftrightarrow \bar{r}\left(x_{i}\right)>\bar{r}\left(x_{j}\right) \Leftrightarrow \sum_{k=1}^{m} r_{k}\left(x_{i}\right)>\sum_{k=1}^{m} r_{k}\left(x_{j}\right) \\
& \Leftrightarrow \sum_{k=1}^{m} \sum_{p=1}^{n} r_{i p}^{k}>\sum_{k=1}^{m} \sum_{p=1}^{n} r_{j p}^{k} \\
& \Leftrightarrow \sum_{k=1}^{m} \sum_{p=1}^{n} N\left(r_{j p}^{k}\right)>\sum_{k=1}^{m} \sum_{p=1}^{n} N\left(r_{i p}^{k}\right) \\
& \Leftrightarrow \sum_{k=1}^{m} \sum_{p=1}^{n} s_{j p}^{k}>\sum_{k=1}^{m} \sum_{p=1}^{n} s_{i p}^{k} \\
& \Leftrightarrow \sum_{k=1}^{m} r_{k}^{\prime}\left(x_{j}\right)>\sum_{k=1}^{m} r_{k}^{\prime}\left(x_{i}\right) \\
& \Leftrightarrow \bar{r}^{\prime}\left(x_{j}\right)>\bar{r}^{\prime}\left(x_{i}\right) \Leftrightarrow x_{j} P^{\prime} x_{i} .
\end{aligned}
$$

\section{Concluding Remarks}

The classic Borda rule has been considered in the literature as an interesting voting system because of its good features and properties. Taking into account this approach or even the generalization 
of the classic Borda rule to the case of individual preferences that are weak orders (rather than linear orders), in this paper we have extended such scheme to the modality of preferences shown by the voters through linguistic labels, as happens in real life. This generalization requires linguistic labels to be added up, and these sums to be ordered. We have proven that this linguistic-based Borda rule shares some interesting properties with the classic one: anonymity, neutrality, consistency, strong monotonicity and unanimity. And, in a more restrictive context, an additional property (also fulfilled by the classic rule) is satisfied as well: reversal symmetry.

It is important to note that the considered linguistic extension of the Borda rule, although presented within a formal framework with an abstract point of view, may become implementable in quite flexible ways. This fact relies on the possibilities of representing linguistic labels by means of different mathematical objects (real numbers, intervals of real numbers, triangular fuzzy numbers, trapezoidal fuzzy numbers, etc.). However, it must be taken into account that the corresponding orderings (in the sets of mathematical objects representing linguistic labels) ought to be compatible with the well-balanced monoid structure. With this caveat assumed, such flexibility should not be viewed as a drawback of our approach. Indeed, to work with vague concepts requires modeling these concepts with appropriate mathematical structures.

\section{Acknowledgements}

The financial support of the Junta de Castilla y León (Consejería de Educación, Projects VA092A08 and GR99), the Spanish Ministerio de Ciencia e Innovación (Project ECO2009-07332) and ERDF are gratefully acknowledged.

\section{References}

1. M. Dummett, Voting Procedures, Clarendon Press, 1984.

2. H. Nurmi, Comparing Voting Systems, D. Reidel Publishing Company, 1987.
3. P. C. Fishburn, "Multiperson decision making: A selective review", in J. Kacprzyk and M. Fedrizzi, (eds.), Multiperson Decision Making Using Fuzzy Sets and Possibility Theory, Kluwer Academic Publishers, 327 (1990).

4. D. Saari, Decisions and Elections. Explaining the Unexpected, Cambridge University Press, 2001.

5. J. Malkevitch, "Mathematical theory of elections", Annals of the New York Academy of Sciences, 607, 8997 (1990).

6. J. C. de Borda, "Mémorie sur les élections au scrutin", in Historie de l'Academie Royale des Sciences, Paris, 1781.

7. P. Gärdenfors, "Positionalist voting functions", Theory and Decision, 4, 1-24 (1973).

8. W. H. Riker, Liberalism against Populism, Waveland Press, 1982.

9. M. Dummett, "The Borda count and agenda manipulation", Social Choice and Welfare, 15, 289-296 (1998).

10. D. Saari, Basic Geometry of Voting, Springer, 1995.

11. D. Black, The Theory of Committees and Elections, Cambridge University Press, 1958.

12. I. McLean and A. B. Urken, Classics of Social Choice, The University of Michigan Press, 1995.

13. T. Marchant, "Valued relations aggregation with the Borda method", Journal of Multi-Criteria Decision Analysis, 5, 127-132 (1996).

14. T. Marchant, "Does the Borda rule provide more than a ranking?", Social Choice and Welfare, 17, 381-391 (2000).

15. J. L. García-Lapresta and M. Martínez-Panero, "Borda count versus approval voting: A fuzzy approach”, Public Choice, 112, 167-184 (2002).

16. J. L. García-Lapresta and M. Martínez-Panero, "A fuzzy Borda count in multi-person decision making", in T. Trzaskalik and J. Michnik, (eds.), Multiple Objective and Goal Programming. Recent Developments, Advances in Soft Computing, Springer, 46-60 (2002).

17. L. A. Zadeh, "The concept of a linguistic variable and its applications to approximate reasoning", Information Sciences, Part I 8, 199-249, Part II 8, 301-357, Part III 9, 43-80 (1975).

18. L. A. Zadeh, "Fuzzy logic = computing with words", IEEE Transactions on Fuzzy Systems, 4, 103-111 (1996).

19. J. L. García-Lapresta, L. L. Lazzari and M. MartínezPanero, "A group decision making method using fuzzy triangular numbers", in C. Zopounidis, P. M. Pardalos and G. Baourakis, (eds.), Fuzzy Sets in Management, Economics and Marketing, World Scientific, 35-50 (2001).

20. J. L. García-Lapresta, B. Llamazares and M. Martínez-Panero, "Linguistic matrix aggregation operators: Extensions of the Borda rule", in B. Reusch, 
(ed.), Computational Intelligence, Theory and Applications, Advances in Soft Computing, Springer, 561575 (2006).

21. F. Herrera, E. Herrera-Viedma and J. L. Verdegay, "A linguistic decision process in group decision making", Group Decision and Negotiation, 5, 165-176 (1996).

22. R. R. Yager, "On the retranslation process in Zadeh's paradigm of computing with words", IEEE Transactions on Systems, Man, and Cybernetics Part B: Cybernetics, 34, 1184-1195 (2004).

23. J. L. García-Lapresta, "A general class of simple majority decision rules based on linguistic opinions", Information Sciences, 176, 352-365 (2006).

24. J. L. García-Lapresta, M. Martínez-Panero and L. C. Meneses, "Defining the Borda count in a linguistic decision making context", Information Sciences, 179, 2309-2316 (2009).

25. D. Black, "Partial justification of the Borda rule", Public Choice, 28, 1-16 (1976).

26. R. R. Yager, "Non-numeric multi-criteria multiperson decision making", Group Decision and Negotiation, 2, 81-93 (1993).

27. V. Torra, "Aggregation of linguistic labels when semantics is based on antonyms", International Journal of Intelligent Systems, 16, 513-524 (2001).

28. A. R. de Soto and E. Trillas, "On antonym and negate in fuzzy logic", International Journal of Intelligent Systems, 14, 295-303 (1999).

29. J. Kacprzyk, S. Zadrózny and Z. W. Raś, "How to support consensus reaching using action rules: A novel approach", International Journal of Uncertainty, Fuzziness and Knowledge-Based Systems, (forthcoming).

30. H. Nurmi, "Approaches to collective decision making with fuzzy preference relations", Fuzzy Sets and Systems, 6, 249-259 (1981).

31. J. L. García-Lapresta and B. Llamazares, "Aggregation of fuzzy preferences: Some rules of the mean", Social Choice and Welfare, 17, 673-690 (2000).

32. M. Delgado, M. A. Vila and W. Voxman, "On a canonical representation of fuzzy numbers", Fuzzy Sets and Systems, 93, 125-135 (1998).

33. D. Dubois and H. Prade, "Fuzzy numbers: An overview", in J. C. Bezdek (ed.), Analysis of Fuzzy Information, vol. 1, CRC Press, 3-39 (1987).

34. F. Herrera and E. Herrera-Viedma, "Linguistic decision analysis: steps for solving decision problems under linguistic information", Fuzzy Sets and Systems, 115, 67-82 (2000).

35. F. Herrera and E. Herrera-Viedma, "Choice functions and mechanisms for linguistic preference relation", European Journal of Operational Research, 120, 144$161(2000)$.

36. H. P. Young, "Social choice scoring functions", SIAM Journal on Applied Mathematics, 28, 824-838 (1975).

37. P. C. Fishburn, "Axioms for approval voting: Direct proof", Journal of Economic Theory, 19, 180-185 (1978).

38. H. P. Young and A. Levenglick, "A consistent extension of Condorcet's election principle", SIAM Journal on Applied Mathematics, 35, 285-300 (1978).

39. K. O. May, "A set of independent necessary and sufficient conditions for simple majority decision", Econometrica, 20, 680-684 (1952).

40. D. Saari, "Explaining all three-alternative voting outcomes", Journal of Economic Theory, 87, 313-355 (1999). 